Project Number 86890

A Hydrologic-Geophysical Method for Characterizing Flow and Transport Processes within the Vadose Zone

$6 / 30 / 04$

RESEARCH OBJECTIVE

The research objective of this project is to analyze the resolution of two different geophysical imaging techniques (electrical resistivity tomography and cross-borehole ground penetrating radar) for monitoring subsurface flow and transport processes within the vadose zone. This is being accomplished through a coupled approach involving large scale unsaturated flow modeling, petrophysical conversion of the resulting hydrologic properties to a geophysical property model and generation of synthetic geophysical data, followed by the inversion of the synthetic geophysical data. The resolution, benefits, and limitations of the geophysical techniques will then be ascertained through analysis and comparison of the images to the original hydrologic model. In addition, increasing levels of complexity will be added to the models as the project progresses through the addition of heterogeneity in the original hydrologic property model, and through uncertainty in the petrophysical relationship that couples the geophysical model to the hydrologic simulation.

\title{
RESEARCH PROGRESS AND IMPLICATIONS:
}

Hydrologic modeling efforts have been based on a constant flux infiltration experiment performed with funding from the original EMSP (project 55332) at the Sandia-Tech Vadose Zone Facility (STVZ) near Socorro New Mexico. This experiment involved infiltrating potable water over a 1-meter square surface area while preventing water infiltration or evaporation from the surrounding area. The infiltration rate was chosen to maintain unsaturated conditions in the underlying heterogeneous layered fluvial deposits. Our modeling strategy has been to start with simple models and incorporate more detail and complexity in an effort to simulate infiltration. Simplistic 2D simulations were performed with 'Hydrus2D' (Simunek van Genuchten, 1999) to explore the effect of grid discretization, initial conditions, and property fields on the simulated results. Information gleamed from these preliminary results were used as a guide in setting up spatially dense 3-D property fields used with POR-SALSA, an algorithm designed to run high resolution simulations on massively parallel machines (See Martinez et al, 1997).

Most of the modeling effort over the last year has been devoted to compiling 3D hydrologic property and running 3-D simulations on POR-SALSA. Early simulations involved one layer-homogeneous property fields with 25, 12.5 and 6.25 grid discretizations within a $1728 \mathrm{~m}^{3}(12 \mathrm{~m}$ on a side) field. More recently simulations have included a 6-layer property field approximating the hydrostratigraphic units observed at the STVZ at the each grid discretizations mentioned above. Initial 6-layer simulations incorporated uniform layers. Currently we are performing simulations using spatially correlated random (non-uniform) property fields within each layer. This suite of results is providing the data necessary for the geophysical related studies in this project while 
also providing the opportunity to investigate of the effects of grid spacing, upscaling, and the effect of a variety of non-uniform property distributions on simulated results.

While hydrologic data were being compiled and the 3D unsaturated flow model constructed and initial simulations were being completed in 3D, 2D flow modeling was conducted which employed 'Hydrus2D' to crudely simulate the pre-infiltration conditions at the STVZ site. No spatial variability was included within the 6 different units employed, and the petrophysical relationship to convert from hydrologic to geophysical properties was assumed to be exact. Cross-borehole GPR synthetic data were calculated using the finite difference time domain (FDTD) scheme given in Holliger and Bergman (2002), and travel-time and amplitude data inverted using the Geotom algorithm (Jackson and Tweeton, 1996; Geotom, 1998).

Three cases were considered to help in the analysis of the field data collected at the STVZ. In case 1, the resolution of the background hydrologic model was analyzed. Water content and attenuation images inverted by curved-ray and straight-ray inversion methods were compared with each other, and it was found that attenuation within the clay layer is overestimated near the boreholes but underestimated between boreholes in the inverted images. On the other hand, velocity/water content images computed from the FDTD results are similar to the input models. This suggests that attenuation images are more sensitive to ray paths than are the velocity/water content images, and the correct physics should be accounted for in the attenuation inversion processes.

In case 2, time-lapse EM models were created from results of the unsaturated flow modeling, and the straight-ray inversion method was employed for both water content and attenuation estimation. Comparing the water-content difference images generated from the FDTD results with the input model shows that the straight-ray inversion is able to resolve the developing water plume in a time-lapse sense, although the radar wave may actually travel along a curved path. On the other hand, the inverted attenuation shows that artifacts manifest as localized regions of high attenuation resulting from the use of straight-ray inversion. This results in the generation of artifacts caused by incorrect straight-ray approximations being applied to the data, or by improper processing for the attenuation data.

Lastly a very crude set of XBGPR models were completed to simulate conductivity anomalies that would be generated during the solute infiltration experiments; these did not involve actual flow modeling and a conversion as before, but rather simple construction of models that contain conductive anomalies mimicking a preferential flow path. Images of the saltwater plume simulations with and without preferential flow paths show that the location of the more saline fluid is fairly well resolved in a time-lapse sense within the attenuation images. In addition, artifacts such as those exhibited in time-lapse images of the transient fluid flow models do not appear in this case. This is likely due to the fact that the ray paths are not changing between the saltwater infiltration and background images. This reinforces the fact that data collected during non-steady state flow conditions, such as those in case 2, are susceptible to inaccuracies if straight ray inversion is employed.

At Multi-Phase Technologies, significant effort has been expended in developing forward modeling strategies for very large meshes. One problem has been the accurate representation of semi-infinite regions using finite-sized meshes. In the past, for meshes with tens of thousands of nodes, these boundaries were approximated using a 
combination of padding cells, large, elongate cells around the central modeling region, and approximate, non-reflective boundary conditions. For large meshes with hundreds of thousands or millions of elements, normal methods of choosing padding cells created numerical instability in the solution that was reflected in decreased accuracy. We found that obtaining optimal accuracy is a tradeoff between reducing boundary effects and maintaining an acceptable condition number for the system of equations in the forward model. Testing has produced rules of thumb that allow accuracies of $1 \%$ or better when comparing to simple analytical models. Using these rules, forward modeling of the STVZ has been carried out to element sizes as small as $12.5 \mathrm{~cm}$ and future work will include models with cells as fine as $6.25 \mathrm{~cm}$.

Modeling carried out on the initial layered and non-layered random models developed from conversion from POR SALSA models carried out at Sandia National Laboratories. Forward models are then inverted using coarse meshes similar to those used in typical field situations. Initial results tend to confirm prior field observations that initial, background inversion, those carried out prior to infiltration, provides a mediocre view of subsurface geology. However, inversions emphasizing change over time such as the as the movement of infiltrating water appear to provide good estimates of these changes and thus a good estimate of the location and path of the infiltrating front.

\section{PLANNED ACTIVITIES}

We are in the process of setting up and running 6-layer uniform and non-uniform simulations at $3.125 \mathrm{~cm}$ grid discretizations to complete our systematic study of grid spacing issues. In the next round of simulations, we will begin to explore methods to make the simulated flow field more closely resemble the observed flow field at the STVZ. This calibration exercise will provide a starting point for the next step where we will use all the available STVZ site characterization information to develop property fields that more closely represent those at the STVZ.

Currently, and in the future we will be extracting 2D cross-sections from the 3D Sandia hydrologic model, calculating cross-well GPR synthetics, and processing and imaging the data. The images will be then compared to both the original model, as well as the ERT images to determine pros and cons in resolving cases of increasing complexity.

Future ERT work will include increasingly complex models with varying amounts of noise added to both the data and the theory relating hydrological and geophysical parameters. Additional work is anticipated in trying to gain a better understanding of the relation between hydrological parameters and geophysical images and to improve the quality of background images.

\section{INFORMATION ACCESS}

Published Peer Reviewed Journal Articles

Tentatively Accepted Peer Reviewed Articles, or Articles under Review

$\underline{\text { Articles in Preparation }}$ 
1. Chang, P. Y., and Alumbaugh, D., 2004, Geophysical image analysis of a series of vadosezone infiltration experiments through coupled unsaturated flow and geophysical modeling; to be submitted to Water Resources Research.

\section{Conference Proceedings}

1. Alumbaugh, D. L, Chang, P. Y., LaBrecque, D., Stueben, M., Brainard,J, and Hall, L., 2003 Investigating vadose-zone flow and transport processes using cross borehole GPR and electrical resistivity; Presented at the 2003 Annual Meeting of the Society of Exploration Geophysicists, October of 2003, Dallas, TX.

2. LaBrecque, D., Sharpe, R and Stubben, M. Towards three and four dimensional data collection for electrical resisitivity data: Proceedings of the Symposium on the Application of Geophysics to Engineering and Environmental Problems.

\section{Theses}

1. Chang, P. Y., 2003, An Analysis Of A Coupled Geophysical Tomography Hydrological Modeling Method To Characterize Hydraulic Properties, And Flow And Transport Process In The Vadose Zone; Thesis submitted to partially satisfy the PhD Degree in Geological Engineering, University of Wisconsin-Madison.

\section{References}

Geotom, 1998, User Mannual for GeotomCG and GeoTom3D, GeoTom, LLC., Apple valley, Minnesota.

Holliger and Bergman, 2002, Numerical modeling of borehole georadar data, Geophysics, Vol 67, No. 4, p. 1249-1257.

Martinez, M. J., P. L. Hopkins, and P. C. Reeves, PorSalsa User's Manual, SAND20011555, Sandia National Laboratories, Albuquerque, New Mexico, 2001.

Simunek, J, Senja, M, and van Genuchten, M. Th., 1999, Manuel for Hydrus2D/Meshgen-2D; Simulating Water Flow and Solute Transport in TwoDimensional Variably Saturated Media, International Ground Water Modeling Center, Colorado School of Mines.

Tweeton, D., 1998, Manual for GeoTomCG; Installing and Running the ThreeDimensional Tomography Program. 